hellgelbe Hemmungszonen sichtbar sind. Die rotbraune Farbe rührt von der Reduktion von Triphenyltetrazoliumsalz ins zugehörige Formazan her. 6-Aminopenicillansäure, welche mikrobiologisch unwirksam ist, wurde auf der Platte vor der Sichtbarmachung ins aktive Benzylpenicillin (Penicillin G) verwandelt. Direkt nachweisbar sind: Penicillin V und $G, 2,6$-Dimethoxybenzylpenicillin, 6-Aminopenicillansäure, Tetracyclin, Anhydrotetracyclin, Hydroxytetracyclin, Chlorotetracyclin, Demethyltetracyclin, Anhydrodemethyltetracyclin, Desoxytetracyclin, Anhydrochlorotetracyclin und Rifomicin B, O, S und SV.

\section{A Simple Detection of Fat-Soluble Vitamins on Alumina Thin Layer-Chromatograms}

For the detection of fat-soluble vitamins after chromatographic separation on thin alumina layer, $70 \%$ perchloric acid and $98 \%$ sulfuric acid proved to be useful. Similar reactions have been previously described ${ }^{1-5}$ and some have even been used for colorimetric determination. A survey of colours given by individual fat-soluble vitamins (Vitamin and provitamins $A$, vitamin $D_{2}, E, K_{1}$, $K_{2}, K_{3}$ ) is listed in the Table. The method is advantageous because of the stability of the reaction products and because alumina itself gives no coloration with the agents mentioned. The application of the acids is performed in such a way that the chromatograms after the develop-

Colour reactions of fat-soluble vitamins with some acids

\begin{tabular}{lll}
\hline Vitamin & $70 \%$ perchloric acid & $98 \%$ sulfuric acid \\
\hline $\mathrm{A}$ & violet & blue violet \\
$\mathrm{D}_{2}$ & orange brown & orange red \\
$\mathrm{E}$ & brown & brown \\
$\mathrm{K}_{1}$ & yellow brown & yellow brown \\
$\mathrm{K}_{2}$ & yellow brown & yellow brown \\
$\mathrm{K}_{3}$ & yellow brown & yellow brown \\
Provitamins A & blue & blue \\
\hline
\end{tabular}

\section{Eine einfache Methode zur Gewinnung von Exuvialflüssigkeit bei der Honigbiene (Apis mellifica L.)}

Die Exuvialflüssigkeit, welche beim Häutungsvorgang der Insekten von den Epidermiszellen in den entstehenden Spaltraum zwischen Matrix und Cuticula abgeschieden wird und durch ihre Fermente (Chitinasen undProteinasen) die alte Endocuticula von innen her auflöst, ist meines Wissens bis jetzt nur bei der Wanze Rhodnius prolixus ${ }^{\mathbf{1}, 2}$, beim Mehlkäfer (Tenebrio molitor) ${ }^{3}$, dem Riesenseidenspinner (Platysamia cecropia) ${ }^{4}$ und beim Maulbeerspinner (Bombyx mori) 5,6 untersucht worden. Der Grund mag wohl der sein, dass die Gewinnung einer genügenden Menge dieses Sekretes für die biochemische Untersuchung im allgemeinen sehr schwierig ist.

Ich bin in der Lage, hier eine einfache Methode zu beschreiben, welche gestattet, bei Arbeiterinnen- und Drohnenlarven der Honigbiene grössere Mengen von Exuvial flussigkeit zu gewinnen. Das Verfahren beruht auf der Beobachtung, dass die normale Verpuppung der Biene nicht nur von der Tätigkeit der endokrinen Organe, son-
Summary. Numerous tetracyclines, penicillins and rifomycins on thin layer chromatograms may be detected by microbiological development with Sarcina lutea and Bacillus subtilis in the presence of triphenyltetrazolium salts. This technique is exceptionally sensitive $(0.01-0.1 \gamma)$, the inhibition zones being much sharper than on paper chromatograms.

B. J.R. Nicolaus, C. Coronelli und A. Binaghi Laboratori di Ricerca della Lepetit S.p.A. Milano (Italien), 22. Juni 1961.

ment are first air-dried and then the acid is made to soak into the alumina layer in a direction perpendicular to that of the previous development (a technique similar to the proper chromatography). The coloration is visual immediately in the wet state, but its intensity decreases slowly $(6 \mathrm{~h})$. This method proved valuable, since no destruction of the alumina layer takes place as in case of the spraying technique.

Zusammentassung. Unter Verwendung von 70prozentiger Perchlorsäure und 98prozentiger Schwefelsäure wurde eine einfache Technik zur Detektion der fettlöslichen Vitamine auf Aluminiumoxyd-Dünnschicht-Chromatogrammen ausgearbeitet.

J. Brattiná and J. Davívek

Central Research Institute of Food Industry, Prague, and Department of Chemistry and Control of Food Institute of Chemical Technology, Prague (Czechoslovakia), March 21. 1961.

1 A. E. Pacini and M. H. Taras, J. Amer. pharm. Assoc. 26, 721 (1937).

2 P. Flesh, Proc. Soc. exp. Biol. Med. 84, 148 (1953).

3 F. CutA and J. CelikovskY, Chem. Listy 48, 1346 (1954).

4 S. Ueno, Ind. Eng. Chem. 7, 596 (1915).

5 E. D. Robin, Science 102, 17 (1945).

dern auch vom Vorhandensein des Larvengespinstes (Kokon) abhängig ist ${ }^{7-10}$. Ohne dieses Gespinst oder eine Unterlage von gleichwertiger Oberflächenbeschaffenheit tritt die letzte Larvenhäutung, das heisst die Häutung der Larve zur Puppe entweder gar nicht ein oder verläuft regelwidrig, so dass höchstens mehr oder weniger verkrüppelte und lebensunfähige Imagines entstehen. Man kann sich davon leicht überzeugen, wenn man ausgewachsene

1 V. B. Wigglesworth, Quart. J. micr. Sci. 76, 270 (1933).

2 V. B. Wigglesworth, The Principle of Insect Physiology (Methuen \& Co. Ltd., London 1950), p. 32.

${ }^{3}$ Ch. Jeuniaux, Arch, int. Physiol. Biochim. 63, 114 (1955).

- J. V. Passonneau und C. M. Williams, J. exp. Biol. Med. 30, 545 (1953).

5 Y. Hamamura, S. Jida und M. Otsuka, Bull. agric. Chem. Soc. Japan $16(1940)$.

${ }^{6}$ Ch. Jeuniaux und M. Amaneu, Arch. int. Physiol. Biochim. 63, 94 (1955); Exper. 11, 195 (1955).

7 A. V. VELICH, Z. wiss. Zool. 136, 210 (1930).

${ }^{8}$ W. FYG, Mitt. Schweiz, entomol. Ges. 29, 317 (1956).

${ }^{9} \mathrm{~W}$. FYG, Insectes sociauX 4, $327(1957)$.

${ }^{10}$ W. FYG, Schweiz. Bztg. 81,387 (1958). 